\title{
Practice Spotlight: Pharmacy Practice in a Correctional Setting
}

\author{
Denise Denning, BScPhm \\ Toronto Jail \\ Ministry of Community Safety \\ and Correctional Services \\ Toronto, Ontario
}

$\mathrm{T}$ he Toronto Jail, known colloquially as the Don Jail, is a maximum-security provincial correctional facility, housing 500 to 600 male inmates who are awaiting trial on charges ranging from minor offences to first-degree murder. The average length of stay is 3 to 6 months, although some inmates may reside in the jail for a year or more while awaiting deportation hearings or dangerous-offender assessments. The Toronto Jail is a unique practice setting for health care providers because clients are housed in the facility for nonmedical reasons.

The health care unit in the Toronto Jail provides most of the inmates' medical needs, including wound care (e.g., stitching of lacerations); diabetes care, such as foot care and blood glucose monitoring; diagnostic tests (e.g., radiography, ultrasonography, and blood work); and the provision of all medications except those in IV dosage forms (which must be administered in hospital). Within the jail, nurses process all medication orders except those for methadone. This system is made possible by a protocol of the Ontario College of Pharmacists, which permits the delegation of dispensing in health care facilities where there is a paucity of pharmacists. ${ }^{1}$ The exception to this system, methadone, must be dispensed by a pharmacist, as required by law. As such, the Toronto Jail pharmacist administers the methadone program, verifying all claims of methadone maintenance with community providers, obtaining physicians' orders for methadone, and preparing the doses. When the pharmacist is away, the nurses fax methadone prescriptions to a retail pharmacy that delivers to the jail.

Most of the inmates are under 40 years of age, and many of them are in good health, but some medical conditions have been found to be more prevalent in inmate populations. Low socioeconomic status, major psychiatric illnesses, homelessness, and substance abuse and dependence are risk factors for incar-

ceration. In a survey performed in 2003 and 2004, Calzavara and others ${ }^{2}$ found that the prevalences of hepatitis $\mathrm{C}$ and HIV infections among inmates in Ontario provincial jails were 22 and 11 times those of the general population, respectively. Institutional surveys have revealed that almost one-quarter of inmates suffer from a major psychiatric illness. Inmates with chronic medical conditions tend to arrive in the jail with a history of poor adherence to their medications or treatment protocols when living in the community. Therefore, consistent access to health care during incarceration results in an improvement in their health status. For example, among inmates with HIV, CD4+ cell counts increase and viral loads decline, inmates with diabetes achieve better glycemic control, and nearly all inmates enjoy generally improved health because they are receiving consistent treatment of both major and minor maladies.

The Ministry of Community Safety and Correctional Services, which funds all medications for inmates in its custody, attempted to phase out the pharmacist position at the Toronto Jail upon the retirement of the previous pharmacist in 1996. However, the increasing numbers of inmates who were taking methadone after the prescribing restrictions on this drug were lifted in 1996, along with the attendant cost of acquiring doses from retail pharmacies, prompted the ministry to reconsider its decision. As a result, the current pharmacist, Denise Denning, was hired in 1997.

Before working at the jail, Ms Denning completed a specialized pharmacy residency at the Centre for Addiction and Mental Health, focusing on the treatment of drug and alcohol abuse. More recently, she completed an online course on buprenorphine for the treatment of opioid dependence. She is responsible for maintaining the supply of medications for the jail, administering the methadone program, and providing pharmaceutical care to inmates with complex medical conditions. She also acts as an addiction medicine resource for the ministry and recently delivered a presentation on the treatment of opioid dependence at a continuing education symposium for nurses in Ontario correctional institutions. Beyond the technical aspects of administering the methadone program, Ms Denning monitors the charts of all inmates who are receiving methadone, checking for potential drug interactions. 
For instance, Ms Denning consults with the jail's physicians whenever they order a benzodiazepine for an inmate who is already taking methadone, as the combination of methadone and a benzodiazepine is a prominent cause of lethal overdose. ${ }^{3}$ She also suggests alternatives to the many medications that exhibit pharmacokinetic interactions with methadone, promoting the judicious use of drugs that potentiate or inhibit cytochrome P450 isozyme 3A4.

When Ms Denning started working at the Toronto Jail, she was informed that the position did not involve contact with inmates and that the jail's pharmacist had traditionally stayed in the health care unit, preparing doses of methadone and ordering medications from wholesalers. Ms Denning suggested to management that expanding the pharmacist's role to include pharmaceutical care would help to improve patient outcomes and control spiralling drug costs. She instituted more rational inventory control measures and also started identifying and reviewing the charts of inmates with more medically challenging situations upon their arrival at the jail, targeting inmates with chronic conditions such as HIV, hypertension, type 1 and 2 diabetes, psychiatric disorders, and other significant but less common conditions, such as organ transplantation. The jail's physicians have appreciated the pharmacist's assistance in determining more rational pharmacotherapy for these individuals. Other examples of newly admitted inmates that Ms Denning has targeted for review are those who have been receiving long-term therapy with proton pump inhibitors in the community and those with prescriptions for drugs that are not usually associated with abuse, dependence, and tolerance, but that are abused in correctional facilities (e.g., bupropion, gabapentin, and quetiapine).

A growing area of prominence in health care in the correctional setting is the treatment of hepatitis C. Ms Denning ensures that inmates for whom pegylated interferon and ribavirin have been prescribed undergo pertinent blood work when warranted. In addition, she acquires filgrastim and erythropoietin, drugs that are not usually kept in the jail's medication inventory but that are used as adjuncts in hepatitis $\mathrm{C}$ treatment, to manage the adverse effects of interferon and ribavarin. For complex cases such as these, Ms Denning consults the literature and health care providers in the community to ensure that she makes appropriate dosing recommendations to the jails' physicians.
Ms Denning is 1 of only 4 pharmacists working in Ontario's provincial correctional facilities. The role of each of these 4 pharmacists is slightly different, reflecting institutional variations and the strengths of each individual. One of the other pharmacists is the senior pharmacy consultant, who delegates dispensing to all nurses in the ministry. The other 2 pharmacists have more of a dispensing role, although all make themselves available for drug-related inquiries from other jails that do not employ pharmacists. Given this diversity of roles, Ms Denning is helping to develop a formulary specific to correctional facilities, to help increase the consistency of care among facilities and to manage drug inventory costs.

Overall, the ministry in recent years has come to recognize the contribution of pharmacists to correctional health care and plans to continue expanding the role for this profession, hiring more pharmacists and pharmacy technicians to enable development of a medication system more consistent with those found in health care facilities.

\section{References}

1. Protocol for delegating dispensing and compounding in health care facilities. Toronto (ON): Ontario College of Pharmacists; 1995 Jun [cited 2011 Aug 14]. Available from: www.ocpinfo.com/client/ocp/ OCPHome.nsf/web/Protocol+for+Delegating+Dispensing $+\&+$ Compounding + in + Health + Care+Facilities

2. Calzavara L, Ramuscak N, Burchell AN, Swantee C, Myers T, Ford P, et al. Prevalence of HIV and hepatitis $\mathrm{C}$ virus infections among inmates of Ontario remand facilities. CMAJ 2007;177(3):257-261.

3. Hillier W. Methadone program: maintenance treatment program standards and clinical guidelines. Toronto $(\mathrm{ON})$ : College of Physicians and Surgeons of Ontario; 2011 Feb [cited 2011 Aug 7]. Available from: www.cpso.on.ca/uploadedFiles/policies/guidelines/methadone/Meth\%20 Guidelines\%20_Oct07.pdf

The Practice Spotlight series highlights the accomplishments of Canadian pharmacists with unique practices in hospitals and related health care settings. If you have a unique or innovative practice, or you know someone else who should be profiled, please submit your contact information to Mary Ensom, Editor of CJHP (cjhpedit@cshp.ca), and one of our Associate Editors will be in touch with you. 